\title{
Activation of Alveolar Macrophages in Lung Injury Associated With Experimental Acute Pancreatitis Is Mediated by the Liver
}

Daniel Closa, PhD, ${ }^{*}$ Luis Sabater, MD, † Laureano Fernández-Cruz, MD, † Neus Prats, PhD, ‡ Emili Gelpí, PhD, ${ }^{*}$ and Joan Roselló-Catafau, $\mathrm{PhD}^{\star}$

From the *Department of Medical Bioanalysis, Institut d'Investigaciones Biomediques August P. Sunyer, Institut d'Investigaciones Biomediques de Barcelona-Consejo Superior de Investigaciones Cientificas, and the †Department of Surgery, Hospital Clinic, University of Barcelona, Barcelona, and the $\ddagger$ Department of Animal Pathology, Veterinary School, Universitat Autonomia de Bellaterra, Spain

\section{Objective}

To evaluate (1) whether alveolar macrophages are activated as a consequence of acute pancreatitis (AP), (2) the implication of inflammatory factors released by these macrophages in the process of neutrophil migration into the lungs observed in lung injury induced by $\mathrm{AP}$, and (3) the role of the liver in the activation of alveolar macrophages.

\section{Summary Background Data}

Acute lung injury is the extrapancreatic complication most frequently associated with death and complications in severe AP. Neutrophil infiltration into the lungs seems to be related to the release of systemic and local mediators. The liver and alveolar macrophages are sources of mediators that have been suggested to participate in the lung damage associated with AP.

\section{Methods}

Pancreatitis was induced in rats by intraductal administration of $5 \%$ sodium taurocholate. The inflammatory process in the lung and the activation of alveolar macrophages were investigated in animals with and without portocaval shunting 3 hours after AP induction. Alveolar macrophages were obtained by bronchoalveolar lavage. The generation of nitric oxide, leukotriene $\mathrm{B}_{4}$, tumor necrosis factor- $\alpha$, and MIP-2 by alveolar macrophages and the chemotactic activity of supernatants of cultured macrophages were evaluated.

\section{Results}

Pancreatitis was associated with increased infiltration of neutrophils into the lungs 3 hours after induction. This effect was prevented by the portocaval shunt. Alveolar macrophages obtained after induction of pancreatitis generated increased levels of nitric oxide, tumor necrosis factor- $\alpha$, and MIP-2, but not leukotriene $B_{4}$. In addition, supernatants of these macrophages exhibited a chemotactic activity for neutrophils when instilled into the lungs of unmanipulated animals. All these effects were abolished when portocaval shunting was carried out before induction of pancreatitis.

\section{Conclusion}

Lung damage induced by experimental AP is associated with alveolar macrophage activation. The liver mediates the alveolar macrophage activation in this experimental model.
Acute pancreatitis (AP) is a multisystem disease with a variable prognosis depending mainly on the systemic manifestations but also on local conditions. Acute lung injury is

Supported by 1996 Research Grant Program of the Hospital Clinic, University of Barcelona.

Correspondence: Daniel Closa, PhD, Molecular Pathology and Biochemistry of Inflammation Unit, IIBB-CSIC, c/ Jordi Girona, 18-24, 08034Barcelona, Spain.

Accepted for publication August 5, 1998. the extrapancreatic complication most frequently associated with the high rates of morbidity and mortality in severe AP. This respiratory dysfunction is indistinguishable, both clinically and pathologically, from the adult respiratory distress syndrome.

In animal models, the adult respiratory distress syndrome can be induced, in addition of AP, by circulating lipopolysaccharide, hyperoxia, reperfusion injury, or hemorrhagic shock. Investigations performed using these models have suggested that polymorphonuclear neutrophils (PMNs) play 
a pivotal role in the development of adult respiratory distress syndrome stimulated by specific proinflammatory mediators. ${ }^{2}$ Irrespective of the initiating factor, activation and aggregation of neutrophils into the lungs seems to be the final common pathway that leads to lung damage. Several mechanisms capable of attracting neutrophils to the lungs have been described. These mechanisms include the activation of the complement system and the generation of alveolar macrophage-derived factors, which promote neutrophil aggregation into the lung capillaries through their chemotactic properties. ${ }^{3,4}$

The capacity of alveolar macrophages to mobilize a large amount of leukocytes and to release secretory products such as cytokines, arachidonic acid metabolites, and nitric oxide (NO) after their activation in the course of different pulmonary inflammatory diseases ${ }^{5}$ suggests that these cells can be involved in the lung damage associated with AP. The activation of alveolar macrophages seems to be regulated by cytokines and inflammatory mediators, which are reportedly generated during the course of AP.

In earlier studies ${ }^{6}$ we reported the importance of the liver in the development of lung damage after AP induction. Using an experimental model of severe AP induced by intraductal administration of sodium taurocholate, the inflammatory process in the lung can be prevented by a portosystemic shunting of blood. This fact indicates that the lung damage induced by AP is related to the passage through the liver of substances released from the damaged pancreas. Therefore, it could be suggested that soluble mediators released by the liver might trigger the inflammatory response in the lung.

In this study, we designed a series of experiments to evaluate (1) whether alveolar macrophages are activated as a consequence of AP, (2) the possible implication of inflam= matory factors (tumor necrosis fäctor- $\alpha$ [TNF], MIP-2, NO, and leukotriene $\mathrm{B}_{4}$ [LTB4]) released by these macrophages in the neutrophil migration into the lung observed in the course of lung injury induced by AP, and (3) the influence of the liver in the activation of alveolar macrophages during this process.

\section{MATERIALS AND METHODS}

\section{Animals and Procedure}

Male Wistar rats (250 to $300 \mathrm{~g}$ ) were used in this study. All studies were performed in accordance with the European Union regulations for experimental animals. Anesthesia was induced with an intraperitoneal injection of $6 \%$ sodium pentobarbital (Sanofi, France) $(0.1 \mathrm{ml} / 100 \mathrm{~g}$ body weight). A midline laparotomy was performed, the biliopancreatic duct was cannulated through the duodenum, and a small bulldog clamp was used to close the hepatic duct. AP was induced by retrograde injection into the biliopancreatic duct of sodium taurocholate $(5 \%)$ in a volume of 0.1 $\mathrm{ml} / 100 \mathrm{~g}$ body weight using an infusion pump. ${ }^{6}$ Control animals received an infusion of saline solution $0.9 \%$.
Portocaval (PC) shunting was performed by mobilizing the portal vein, ligating its coronary venous tributary, and partially freeing the cava vein above the entrance of the renal vein. Then, blood vessel clamps were placed across the cava vein and the portat vein. An elliptical opening was made on the cava vein, the portal vein was cut after ligation of the end proximal to the liver, and an anastomosis was performed with a 7-0 braid silk attached to two circle taped needles. After removal of the clamps, oozing was readily controlled by a few moments of slight pressure with a sponge. The portal vein was completely occluded for 10 to 12 minutes. $^{7}$

\section{Experimental Design}

In the first series of experiments, the role of the liver in the recruitment of PMNs into the lung during AP was tested in the following groups $(\mathrm{n}=8$ for each group):

1. Control: infusion of saline into the biliopancreatic duct

2. AP: infusion of $5 \%$ sodium taurocholate into the pancreatic duct

3. PC shunting: PC shunting performed immediately before saline infusion into the pancreatic duct

4. PC + AP: PC shunting performed immediately before taurocholate infusion into the pancreatic duct.

To evaluate the inflammatory process in the lung induced by AP, plasma and lung tissue samples were obtained 3 hours after induction, immediately frozen, and maintained at $-80^{\circ} \mathrm{C}$ until assayed. In lung samples, myeloperoxidase (MPO) activity was analyzed. In plasma, lipase activity was measured. In addition, pancreas, liver, and lung samples were obtained for histologic examination.

In a second series of experiments, the activation of alveolar macrophages during the course of AP and the influence of the liver in this activation were evaluated, with the animals distributed in the same four groups $(n=6$ per group) as in the previous series. In this case, 3 hours after the surgical procedure, alveolar macrophages were isolated through bronchoalveolar lavage. Cells were cultured for 24 hours and the levels of NO, LTB4, TNF, and MIP-2 were measured in the supernatants of the culture medium. In addition, the proinflammatory effect of these supernatants was evaluated by instillation of an aliquot of culture medium into the lungs of untreated animals, evaluating neutrophil infiltration by measuring MPO activity.

\section{Assays}

Plasma lipase was determined by using commercial kits (Boehringer Mannheim, Germany), according to the supplier's specifications. MPO was measured photometrically using $3,3^{\prime}, 5,5^{\prime}$-tetramethylbenzidine as a substrate. ${ }^{8}$ Samples were macerated with $0.5 \%$ hexadecyltrimethylammonium bromide in $50 \mathrm{mM}$ phosphate buffer (pH 6). Homog- 
enates were then disrupted for 30 seconds using a Labsonic (B. Braun, Melsungeu, Germany) sonicator at $20 \%$ power and subsequently snap-frozen in dry ice and thawed on three consecutive occasions before a final 30 -second sonication. Samples were incubated at $60^{\circ} \mathrm{C}$ for 2 hours and then spun at $4000 \mathrm{~g}$ for 12 minutes. Supernatants were collected for MPO assay. Enzyme activity was assessed photometrically at $630 \mathrm{~nm}$. The assay mixture consisted of $20 \mu \mathrm{l}$ supernatant, $10 \mu \mathrm{l}$ tetramethylbenzidine (final concentration 1.6 $\mathrm{mM}$ ) dissolved in DMSO, and $70 \mu \mathrm{H} \mathrm{H}_{2} \mathrm{O}_{2}$ (final concentration $3 \mathrm{mM}$ ) diluted in $80 \mathrm{mM}$ phosphate buffer (pH 5.4). An enzyme unit is defined as the amount of enzyme that produces an increase of 1 absorbance unit per minute.

Total protein concentration in homogenates was determined using a commercial kit (BioRad, Munich, Germany).

For the histologic study, samples of lung, liver, and pancreas were obtained, fixed in $10 \%$ neutral buffered formalin, paraplast-embedded, cut into $5-\mu \mathrm{m}$ sections, and stained with hematoxylin-eosin according to standard procedures. Sections were evaluated by light microscopy by two independent observers.

Alveolar macrophages were obtained by bronchoalveolar lavage. Lungs were dissected free of the thoracic cavity and a small length of tubing was inserted into the trachea and ligated. Lavage was carried out using $8 \mathrm{ml}$ of Hank's saline solution instilled four times and withdrawn from the lungs. Lavage fluids from two or three animals were pooled, the cell suspension was centrifuged at $400 \mathrm{~g}$, and the pellet was resuspended in RPMI 1640 medium in the presence of penicillin (100 units $/ \mathrm{ml})$ and streptomycin $(100 \mathrm{mg} / \mathrm{ml})$. Cells were counted, cultured in six dish plates $\left(10^{6} \mathrm{cell} /\right.$ well), incubated for 1 hour at $37^{\circ} \mathrm{C}$ under $5 \% \mathrm{CO}_{2}$ in air, and then washed twice with warm medium to remove nonadherent cells. Supernatants of cultured alveolar macrophages were obtained after 24 hours of cell culture at $37^{\circ} \mathrm{C}$ under $5 \% \mathrm{CO}_{2}$ in air. ${ }^{9}$

The generation of NO from cultured alveolar macrophages was evaluated by measuring the levels of nitrite and nitrate in supernatants of cell culture. ${ }^{10}$ Nitrate was reduced to nitrite with 0.5 units of nitrate reductase in the presence of $50 \mu \mathrm{M}$ NADPH and $5 \mu \mathrm{M}$ FAD. The excess of NADPH was oxidized in the presence of $0.2 \mathrm{mM}$ pyruvate and $1 \mu \mathrm{g}$ lactate dehydrogenase. Nitrite was determined with Greiss reagent by adding $1 \mathrm{mM}$ sulfanilic acid and $100 \mathrm{mM} \mathrm{HCl}$ and $50 \mu \mathrm{l}$ naphthylenediamine. The absorbance was read at $595 \mathrm{~nm}$ and compared with a standard of $\mathrm{NaNO}_{2}$.

Generation of rat TNF and MIP-2 in alveolar macrophage culture supernatants was quantitatively measured by a commercial solid phase sandwich enzyme-linked immunosorbent assay (Biosource International, Camarillo, CA), according to the supplier's specifications.

For the analysis of LTB4, samples of macrophage culture supernatants were processed through preactivated C18 solid phase cartridges (Waters Associates, Milford, MA). Cartridges were washed with $\mathrm{H}_{2} \mathrm{O}(\mathrm{pH} 4)$, and retained eicosanoids were eluted with $4 \mathrm{ml}$ methanol. Methanol extracts

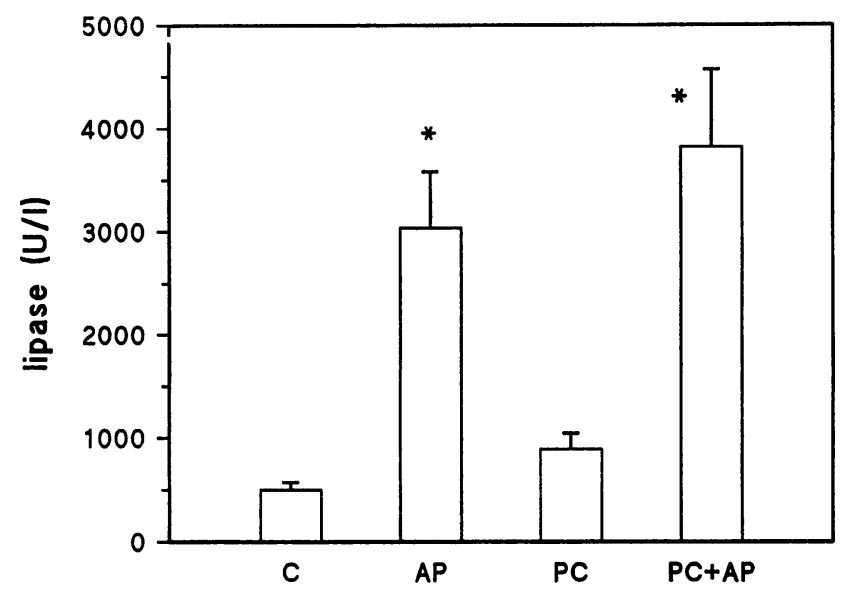

Figure 1. Lipase levels in plasma 3 hours after pancreatitis induction. $\mathrm{C}=$ control; $\mathrm{AP}=$ acute pancreatitis; $\mathrm{PC}=$ portocaval shunting; $\mathrm{PC}+$ $A P=$ portocaval shunting and acute pancreatitis. " $p<0.05$ vs. control.

containing eicosanoids were evaporated to dryness in a centrifugal rotary evaporator, and the residues were resuspended with $500 \mu \mathrm{l}$ of $100 \mathrm{mM}$ Tris- $\mathrm{HCl}$ buffer $(\mathrm{pH} 7.4)$ for subsequent radioimmunoassay measurements by using specific antisera. ${ }^{11}$

To assess the proinflammatory activity of alveolar macrophage-conditioned medium, $500 \mu \mathrm{l}$ of supernatants of alveolar macrophages cultured for 24 hours were collected and instilled into the lungs of untreated rats $(n=6$ per group) using an intratracheal cannula. Lung samples were collected 45 minutes after instillation, and MPO activity was measured. In addition, the effect of the instillation of $500 \mu \mathrm{l}$ of nonconditioned RPMI 1640 medium was evaluated.

\section{Statistical Analysis}

Data are expressed as means \pm standard error of the mean. The means of different groups were compared using a one-way analysis of variance. Student's $t$ test was performed to evaluate significant differences between groups. Differences were assumed to be significant at $\mathrm{p}<0.05$.

\section{RESULTS}

\section{Plasma Lipase Levels}

Figure 1 shows the plasma levels of lipase. AP induction was confirmed by the increased lipase levels found in the AP group. Similar values were obtained in the PC + AP group, indicating a similar level of pancreatic damage. PC shunting without AP induction was not associated with increased levels of plasma lipase; results were similar to those of the control group.

\section{Pulmonary Inflammation}

Neutrophil infiltration into the lungs was evidenced by the increase in MPO activity (Fig. 2). AP induction was 


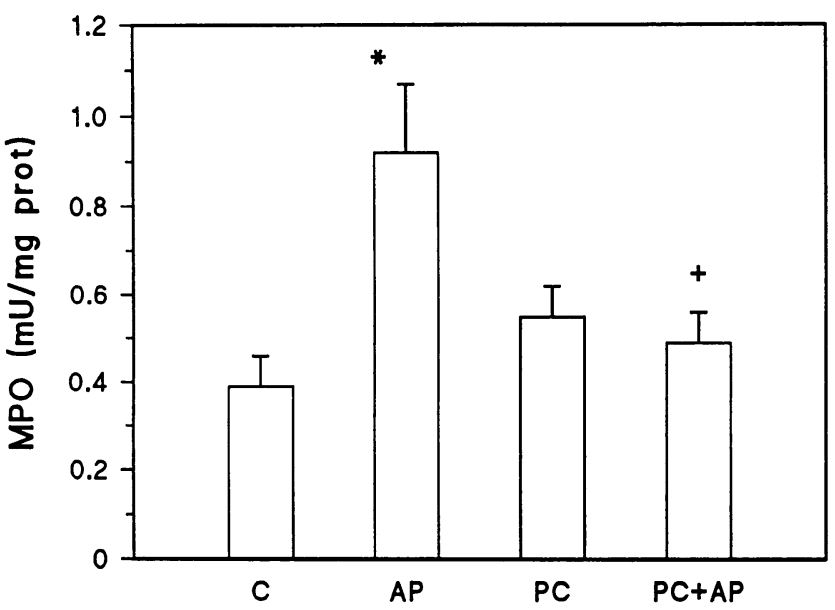

Figure 2. Myeloperoxidase activity into the lungs 3 hours after pancreatitis induction. " $p<0.05 v s$. control; $+p<0.05 v s$. acute pancreatitis group.

associated with significantly increased levels of MPO in lung tissue. This increase was prevented when PC shunting was performed.
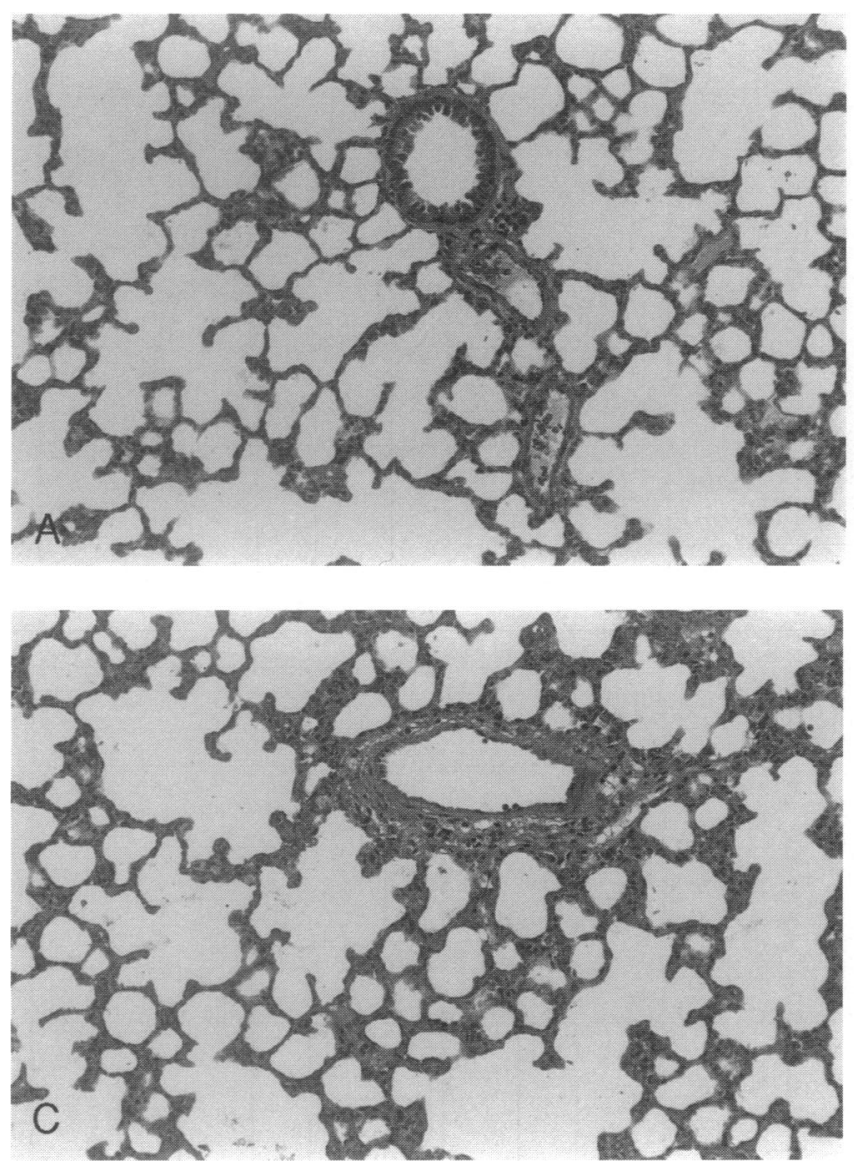

\section{Histologic Evaluation}

The histopathologic study of the lungs (Fig. 3) revealed the main lesions in the AP group. In this group a moderate thickening of the alveolar walls and inflammatory infiltration of PMNs was observed. Moderate hyperemia of the alveolar walls was also present. In the control and PC groups, no significant lesions were observed in the lung parenchyma; only a mild hyperemia of pulmonary alveolar septa was observed. The PC + AP group showed a minimal inflammatory infiltrate in the alveolar walls consisting of scarce PMNs and a mild hyperemia of alveolar septa.

Histopathologic study of the pancreas (Table 1) in the AP group revealed extensive necrosis of the pancreatic tissue, interstitial edema, and PMN adherence to vascular endothelial cells. In the PC + AP group, the same lesions seen in the AP group were observed. The control and PC groups showed only a moderate interstitial edema.

Histopathologic study of the liver (see Table 1) revealed small focal areas of incipient necrosis of hepatocytes in the $\mathrm{PC}$ and PC + AP groups.
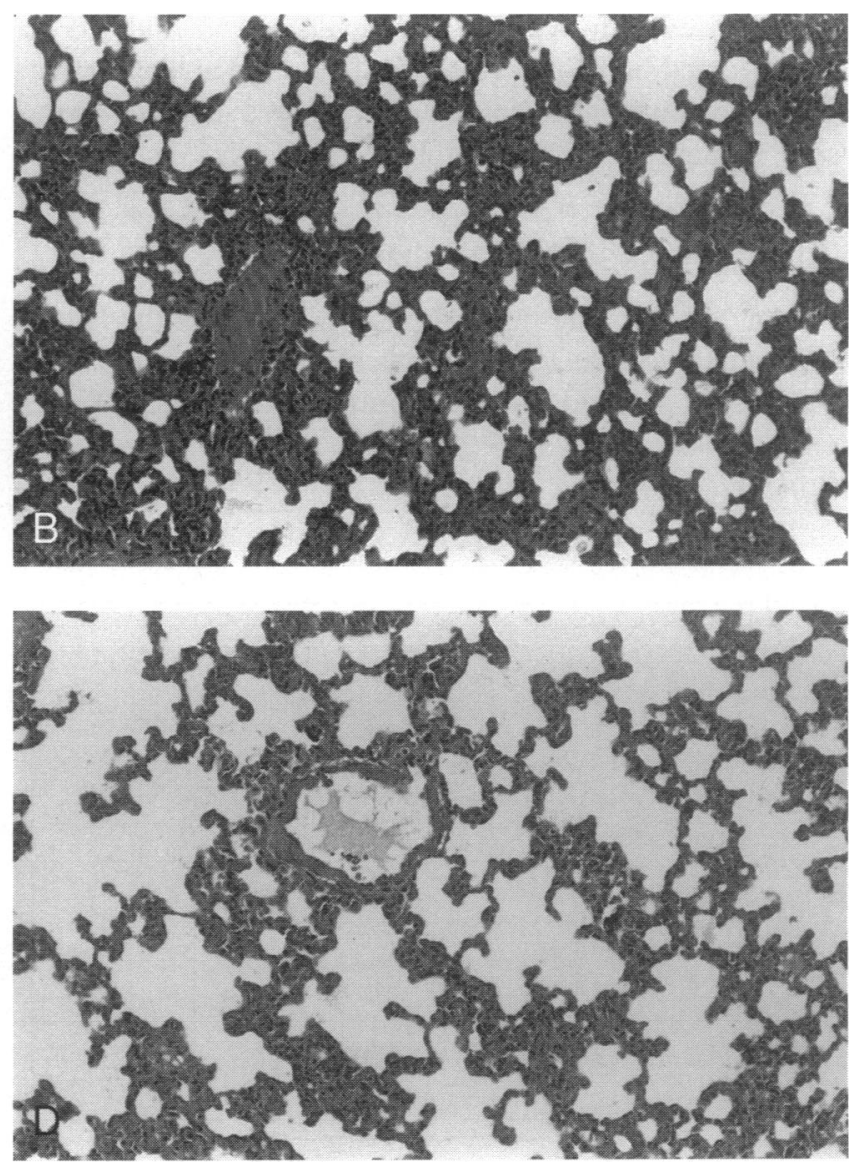

Figure 3. Light microscopy appearance of pulmonary tissue $(\times 20)$. (A) Control group: no lesions in the lung parenchyma. (B) Acute pancreatitis group: moderate thickening of the alveolar walls, polymorphonuclear neutrophil inflammatory infiltration. (C) Portocaval shunt group: no lesions in the lung parenchyma. (D) Portocaval shunt and acute pancreatitis group: minimal thickening of the alveolar walls and scarce presence of polymorphonuclear neutrophils. 
Table 1. HISTOPATHOLOGIC LESIONS OBSERVED IN LUNG, PANCREAS, AND LIVER

\begin{tabular}{|c|c|c|c|c|c|c|}
\hline \multirow[b]{2}{*}{ Group } & \multicolumn{2}{|c|}{ Lung } & \multirow{2}{*}{ 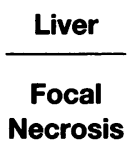 } & \multicolumn{3}{|c|}{ Pancreas } \\
\hline & $\begin{array}{l}\text { PMN Septal } \\
\text { Infiltration }\end{array}$ & Hyperemia & & $\begin{array}{l}\text { Interstitial } \\
\text { Edema }\end{array}$ & $\begin{array}{l}\text { Acinar } \\
\text { Necrosis }\end{array}$ & $\begin{array}{l}\text { PMN Vascular } \\
\text { Adhesion }\end{array}$ \\
\hline Control & - & + & - & + & - & - \\
\hline $\mathrm{AP}$ & ++ & ++ & $+/-$ & ++ & +++ & ++ \\
\hline PC & - & + & - & + & - & - \\
\hline$P C+A P$ & $+1-$ & + & $+1-$ & ++ & +++ & ++ \\
\hline
\end{tabular}

\section{Nitric Oxide Generation}

The effect of AP on the alveolar macrophage activation, evaluated as NO generation, is depicted in Figure 4. These cells showed increased generation of $\mathrm{NO}$ as a consequence of pancreatitis, and this increase was abolished by PC shunting.

\section{Cytokine Generation}

As occurred with NO, increased levels of TNF and MIP-2 were observed in the supernatants of cultured alveolar macrophages obtained after AP induction. These increases were also prevented with PC shunting (Fig. 5).

\section{5-Lipoxygenase Metabolism}

In contrast with the results obtained regarding the levels of NO and cytokines, alveolar macrophage 5-lipoxygenase metabolism remained unmodified after AP induction. There were no changes in LTB4 generation as a consequence of AP induction (Fig. 6).

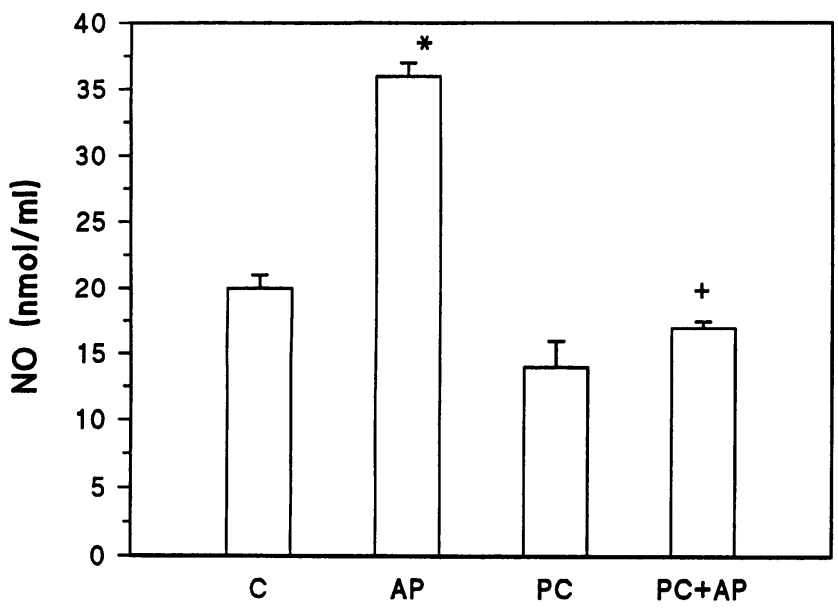

Figure 4. Nitric oxide generation by alveolar macrophages measured as nitrite and nitrate levels. Cells were cultured for 24 hours. Results are the mean of three experiments carried out pooling lavage fluid from two animals. " $p<0.05$ vs. control; $+p<0.05$ vs. acute pancreatitis group.

\section{Chemotactic Activity}

The proinflammatory effect of the instillation into the lung of $500 \mu \mathrm{l}$ supernatant of culture medium obtained after 24 hours of cell incubation is depicted in Figure 7. Instillation of conditioned medium from the control group had no effect on MPO activity of the lungs. In contrast, the instillation of conditioned medium from alveolar macrophages obtained after AP induction induced the accumulation of neutrophils, reflected by increased MPO activity of the
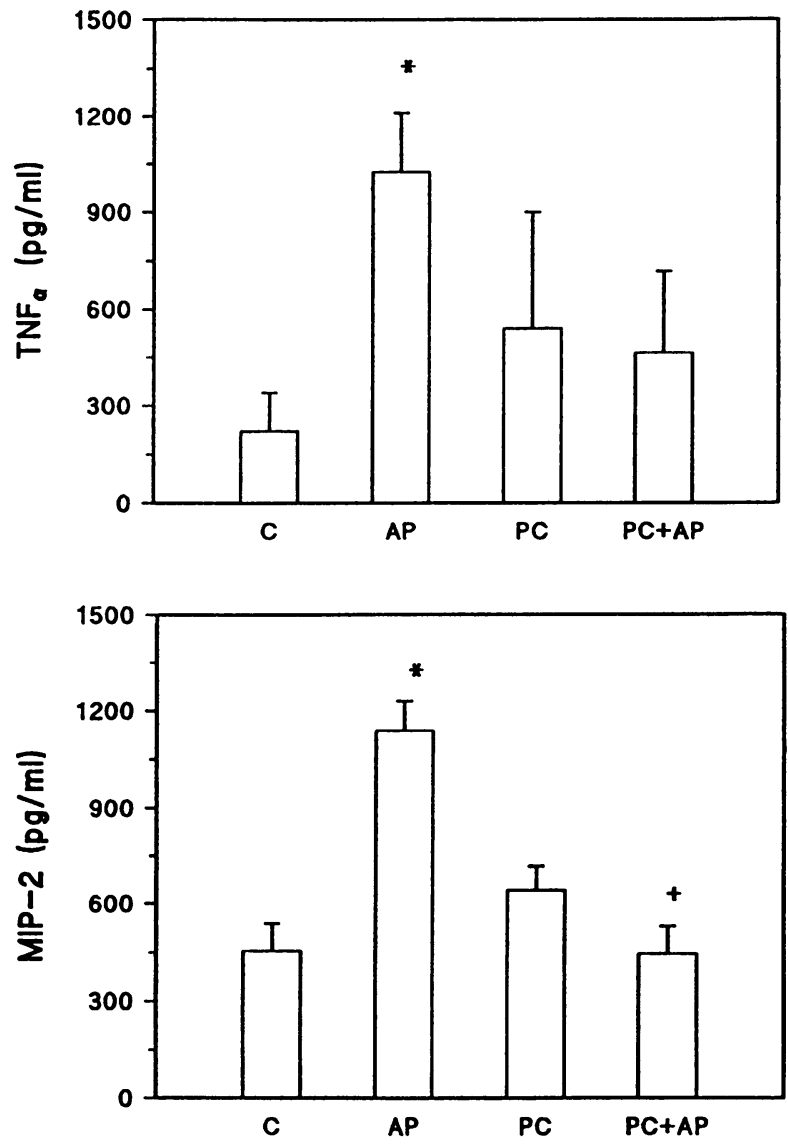

Figure 5. Tumor necrosis factor- $\alpha$ and MIP-2 generation from alveolar macrophages. Cells were cultured for 24 hours. " $p<0.05$ vs. control; $+p<0.05$ vs. acute pancreatitis group. 


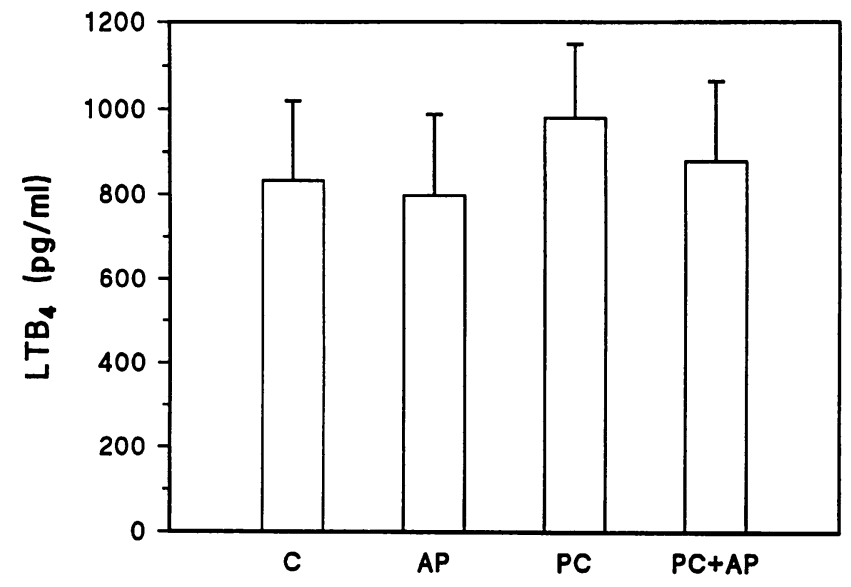

Figure 6. Leukotriene $B_{4}$ levels from alveolar macrophages. Cells were cultured for 24 hours.

lungs. This effect did not appear when PC shunting was carried out before AP induction.

\section{DISCUSSION}

The adult respiratory distress syndrome contributes to the morbidity and mortality rates during AP in approximately one third of patients. ${ }^{12}$ The pathogenesis of lung injury secondary to AP is complex and probably involves multiple mechanisms, such as activated pancreatic proteases, phospholipase A2, and activated complements. ${ }^{13,14}$ It is known that neutrophil accumulation in the lungs is causally involved in the development of lung injury, ${ }^{15}$ but the final mechanism responsible for this neutrophil accumulation is still unknown. In this sense, several experimental models of pulmonary inflammation have shown that alveolar macrophages release chemotactic factors that can promote neutrophil aggregation in the lung capillaries in response to different stimuli. $^{16}$

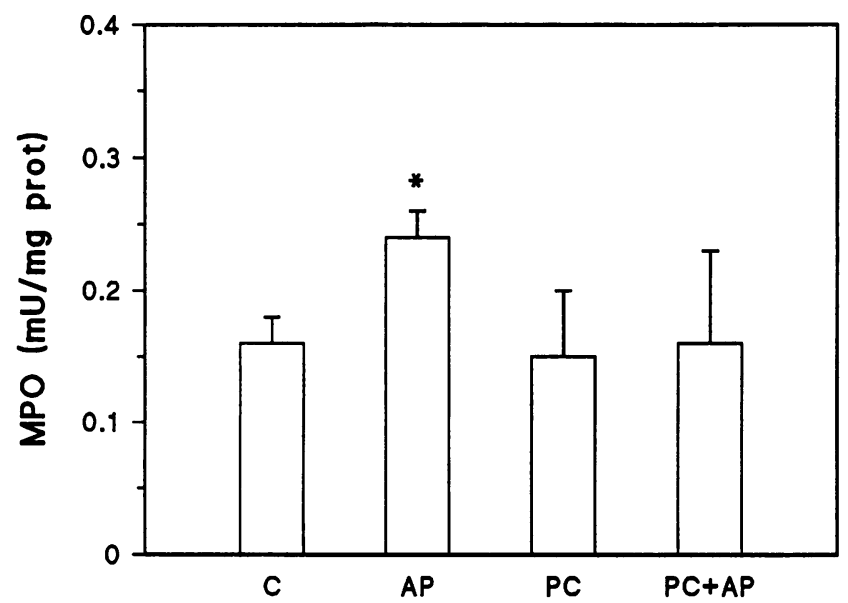

Figure 7. Myeloperoxidase activity in lungs of animals 45 minutes after instillation of $500 \mu \mathrm{l}$ alveolar macrophages-conditioned medium from different experimental groups. Groups indicate the animals from which alveolar macrophages were obtained. ${ }^{*} p<0.05$ vs. control.
In our experimental model, lung inflammation was observed 3 hours after AP induction. This was reflected by the increased MPO activity, indicating the existence of neutrophil accumulation. In accordance with previous works, ${ }^{6}$ PC shunting was found to exert a profound effect on the pulmonary inflammatory process. In our study, this effect was evidenced by the prevention of increases in MPO levels in the lungs, reflecting the absence of neutrophil recruitment (see Figs. 2 and 3). However, PC shunting did not modify the severity of local pancreatic damage, reflected by the similar values of plasma lipase (see Fig. 1) and histologic features (see Table 1).

Taking into account the fact that alveolar macrophages may release secretory products (NO, TNF, MIP-2, LTB4) that can act as proinflammatory agents, we measured these mediators in the supernatants of cultured macrophages obtained after AP induction. Our results show that these cells are activated as a consequence of AP, resulting in an increased generation of NO and cytokines but not LTB4. The ability of alveolar macrophages to generate NO in response to different stimuli is well known. The generation of NO by alveolar macrophages has been reported after exposure of animals to endotoxin, silica, or ozone, or after the activation of these cells by bacteria or cytokines. ${ }^{17}$ Nevertheless, the overproduction of NO may be significant not only in tissue injury, but also in the healing process. Figure 4 shows the increases observed in NO generation after AP induction. This result is in accordance with a previous report by Tsukahara et $\mathrm{al}^{16}$ showing the induction of NO synthase in alveolar macrophages in an experimental model of pancreatitis induced by duct ligation.

Cytokines have been reported to be involved in the development of severe AP. ${ }^{18}$ MIP-2 is a chemotactic cytokine (chemokine) that has been shown to elicit a neutrophil inflammatory response when injected subcutaneously in mice. ${ }^{4}$ This chemokine has a minimal or no effect on neutrophil oxidant production. However, TNF contributes to the inflammatory process by priming neutrophils to generate oxygen free radicals. ${ }^{19}$ LTB4 is a potent chemotactic arachidonic acid metabolite generated by different inflammatory cells, including alveolar macrophages, related to lung injury in different experimental models. ${ }^{20}$

In the present study, although MIP-2 and TNF generation by alveolar macrophages was increased after AP induction (see Fig. 5), LTB4 generation did not change (see Fig. 6). This result suggests that 5-lipoxygenase arachidonic acid metabolism is not involved in the inflammatory process of the lung secondary to AP. On the contrary, it can be suggested that the increased concentrations of MIP-2 and TNF locally generated by alveolar macrophages are involved in the neutrophil infiltration and activation into the lungs.

The liver seems to play a pivotal role in the activation of alveolar macrophages, suggested by the lack of response of these cells when the portal blood was diverted from the liver before AP induction. In the PC + AP group, the absence of alveolar macrophage activation was evidenced by the fact 
that generation of MIP-2, TNF, and NO remained at the same levels as the control group (see Figs. 4 and 5). It could be hypothesized that the absence of chemotactic mediators generated by alveolar macrophages was related to the decreased neutrophil infiltration found in the lungs when PC shunting was carried out before AP induction (see Fig. 2).

Consequently, we examined the ability of the products released from alveolar macrophages to induce chemotaxis for neutrophils into the lungs. Instillation of supernatants from the alveolar macrophages cultured for 24 hours induced infiltration of leukocytes only when these macrophages were obtained after AP induction (see Fig. 7). However, supernatants from control groups and from the PC + AP group were not able to induce the recruitment of neutrophils into the lungs.

The sequence of events that leads to leukocyte infiltration into the lungs secondary to AP involves different physiopathologic mechanisms. ${ }^{21}$ Activation of macrophages may be only one of these pathways. Expression of adhesion molecules into the endothelial cells, synthesis of plateletactivating factor, ${ }^{22}$ and activation of the complement sys$t^{1 e m}{ }^{13}$ are events related to lung injury that probably occur simultaneously with macrophage activation; therefore, it is difficult to establish the exact sequence of events. In addition, the role of the liver in mediating the inflammatory process in the lung remains unclear. Nevertheless, the inhibitory action of PC shunting in the activation of alveolar macrophages indicates that the liver could generate different soluble mediators that activate alveolar macrophages during the course of experimental AP. The nature of these soluble mediators remains to be investigated.

In conclusion, this study shows that lung damage induced by experimental AP develops with alveolar macrophage activation. The liver plays an active role in the activation of alveolar macrophages in this experimental model. In addition, neutrophil recruitment into the lungs during AP seems to be mediated by chemotactic mediators (TNF and MIP-2) released by activated alveolar macrophages.

\section{References}

1. Steinberg W, Tenner S. Acute pancreatitis. N Engl J Med 1994; 330:1198-1210.

2. McCord JM, Gao B, Leff J, Flores SC. Neutrophil-generated free radicals: possible mechanisms of injury in adult respiratory distress syndrome. Environ Health Perspect 1994; 102:57-60.
3. Hohn DC, Meyers AJ, Gherini SC, et al. Production of acute pulmonary injury by leukocytes and activated complement. Surgery 1980; 88:48-57.

4. Driscoll KE. Macrophage inflammatory proteins: biology and role in pulmonary inflammation. Exp Lung Res 1994; 20:473-490.

5. Kienast K, Knorst M, Müller-Quernheim J, Ferlinz R. Modulation of IL-1, IL-6, IL-8, TNF $\alpha$, and TGF- $\beta$ secretions by alveolar macrophages under $\mathrm{NO}_{2}$ exposure. Lung 1996; 174:57-67.

6. Closa D, Bardají M, Hotter G, et al. Hepatic involvement in pancreatitis-induced lung damage. Am J Physiol 1996; 270:G6-G13.

7. Lee SH, Fisher B. Portocaval shunt in the rat. Surgery 1961; 50:667668.

8. Trush MA, Egner PA, Kensler TW. Myeloperoxidase as a biomarker of skin irritation and inflammation. Fd Chem Toxicol 1994; 32:143-147.

9. Collan Y, Kosma VM. Dust toxicity in rat alveolar macrophage cultures. In O'Hare S, Atterwill CK, eds. Methods in molecular biology, vol 43: In vitro toxicity testing protocols. Totowa, NJ: Humana Press; 1994:43-49.

10. Hortelano S, Genaro A, Boscá L. Phorbol esters induce nitric oxide synthase activity in rat hepatocytes. J Biol Chem 1992; 267:24937-24940.

11. Closa D, Roselló-Catafau J, Hotter G, et al. Cyclooxygenase and lipoxygenase metabolism in sodium taurocholate induced acute hemorrhagic pancreatitis in rats. Prostaglandins 1993; 45:315-322.

12. Dugernier T, Reynaert MS, Deby-Dupont G, et al. Prospective evaluation of thoracic-duct drainage in the treatment of respiratory failure complicating severe acute pancreatitis. Intensive Care Med 1989; 15:372-378.

13. Perez HD, Horn JK, Ong R, Goldstein IM. Complement (C5)-derived chemotactic activity in serum from patients with pancreatitis. J Lab Clin Med 1983; 101:123-129.

14. Grönroos JM, Nevalainen TJ. Increased concentrations of synovialtype phospholipase A2 in serum and pulmonary and renal complications in acute pancreatitis. Digestion 1992; 52:232-236.

15. Guice KS, Oldham KT, Caty MG, Johnson KJ, Ward PA, et al. Neutrophil-dependent, oxygen-radical-mediated lung injury associated with acute pancreatitis. Ann Surg 1989; 210:740-747.

16. Tsukahara Y, Horita Y, Anan K, Morisaki T, Tanaka M, Torisu M. Role of nitric oxide derived from alveolar macrophages in the early phase of acute pancreatitis. J Surg Res 1996; 66:43-50.

17. Gorbunov N, Esposito E. Nitric oxide as a mediator of inflammation. Int J Immunopathol Pharmacol 1993; 6:67-75.

18. Norman JG, Franz MG, Fink G, et al. Decreased mortality of severe acute pancreatitis following proximal cytokine blockade. Ann Surg 1995; 221:625-634.

19. Yee J, Christou NV. The local role of tumour necrosis factor in the modulation of neutrophil function at sites of inflammation. Arch Surg 1994; 129:1249-1255.

20. Yoshimura $\mathrm{K}$, Nakagawa $S$, Koyama $S$, et al. Leukotriene $B_{4}$ induces lung injury in the rabbit: role of neutrophils and effect of indomethacin. J Appl Physiol 1993; 74:2174-2179.

21. Guice KS, Oldham KT, Johnson KS, et al. Pancreatitis-induced acute lung injury. Ann Surg 1988; 208:71-77.

22. Zhou W, McCollum MO, Levine BA, Olson MS. Role of plateletactivating factor in pancreatitis-associated acute lung injury in the rat. Am J Pathol 1992; 140:971-979. 